\title{
Analysis of the geometry of a holographic memory setup
}

\author{
A. Márquez ${ }^{1,3}$, E. Fernández ${ }^{2,3}$, F. J. Martínez ${ }^{1}$, S. Gallego ${ }^{1,3}$, \\ M. Ortuño ${ }^{1,3}$, A. Beléndez ${ }^{1,3}$, I. Pascual ${ }^{2,3}$ \\ ${ }^{1}$ Dept. de Física, Ing. de Sistemas y T. Señal, Univ. de Alicante, Ap. 99, E-03080, Alicante, Spain \\ ${ }^{2}$ Dept. de Óptica, Farmacología y Anatomía, Univ. de Alicante, Ap. 99, E-03080, Alicante, Spain \\ ${ }^{3}$ I.U. Física Aplicada a las Ciencias y las Tecnologías Univ. de Alicante, Ap. 99, E-03080, Alicante, \\ Spain
}

\begin{abstract}
Typically 4-f systems are considered as the basis for holographic memory setups. However, other geometries, such as the convergent correlator, may also be considered. This is a setup widely used in optical processing architectures but not so much explored in holographic data storage systems. It provides some benefits when used in optical processing such as flexibility in the adjustment between Fourier filter dimensions and the Fourier transform of the scene. It also allows a wider freedom in the choice of the optical systems (lenses) used in the setup since it is no longer necessary that their focal lengths match, and the total length of the setup may be shortened. In this paper we make use of Fourier optics techniques to analyze the validity and possible benefits of this setup in its application to holographic memories. We consider the recording and the reconstruction steps. Both analytical expressions and simulated results are given.
\end{abstract}

Keywords: holographic data storage, imaging system, optical processing, Fourier optics, liquid crystal display

\section{INTRODUCTION}

Holographic data storage has been a promising and very appealing technology since the first laser developments in the sixties $^{1}$. In the last decade a renewed interest aroused mainly due to the maturing in a series of technologies, such a CCD and CMOS cameras and spatial light modulators, necessary for an optoelectronic system with such high demanding specifications $^{2-7}$. Furthermore two-dimensional memory technologies like CD-ROM, DVD and Blu-Ray have reached the limits of their capacity, and so there is a need for new technological systems capable of storing more information. Holographic data storage is intrinsically a parallelised volume storage and retrieval technology. In this sense is a true 3-D technology which is a new memory technology paradigm if compared with the ones present in the market. It allows an important number of bits to be stored in a recording material with greater capacity, higher density, and faster readout rates than 2-D technology, especially when compared with other optical technologies. Furthermore, it allows for associative memory retrieval of information, which is a basically different memory search capability when compared with present memory technologies ${ }^{8}$. Nowadays the most promising market is possibly the one related with long time archival storage.

In the holographic recording process, the interference between the object beam, carrying the data page typically introduced by a spatial light modulator, and the reference beam is produced. A series of multiplexing techniques ${ }^{9}$ have been proposed enabling to take advantage of the 3-D nature of the holographic process to increase the storage density. When the information needs to be recovered, the reference beam, now called reconstruction beam illuminates the memory and diffraction generates the object beam which can be eventually captured with some camera system. In some multiplexing schemes it is also possible to use the memory system as an associative memory, thus enabling for contentaddressable capability. Basically, no matter the technology developments, most of the architectures proposed for holographic memory systems are based on the so-called 4-f system. This is an optical system typically found in optical processing applications ${ }^{10,11}$. It basically allows for exact cancellation of quadratic phase terms arising in the free space beam propagation. Furthermore, the theoretical expressions describing wavefront propagation to the various planes of

Optical Modelling and Design II, edited by Frank Wyrowski, John T. Sheridan, Jani Tervo, Youri Meuret, Proc. of SPIE Vol. 8429, 84291Y · (c) 2012 SPIE · CCC code: 0277-786X/12/\$18 · doi: 10.1117/12.921294 
interest are easy to calculate, thus probably providing a more intuitive understanding of the performance and suitability of these setups in non-optical processing applications, such as holographic data storage.

In this paper, our aim is to present a different optical architecture, the so-called convergent or VanderLugt correlator, which provides a series of benefits in optical signal processing, basically easy scaling of the dimensions of the Fourier transform of the scene to the scale of the filter in the Fourier plane ${ }^{11}$. This is of no particular interest in holographic data storage systems, but there are other properties which may be of interest, such as the flexibility provided to change the size of the Fourier transform of the data page into the recording material, simply shifting axially the plane of the data page, without the need to change lenses in the system. This enables to increase the areal density of the pages stored in the memory and relaxes the requirements of distances and focal lengths when compared with the 4-f system. In the following sections we develop the necessary analytical expressions, using the well known Fourier optics formalism ${ }^{10,11}$, showing the analytical results in the recording plane and also in the reconstruction process. In all these developments we consider the finite dimension of the Fourier transform in the material, by means of a stop located in front of the recording plane. Some simulations are also provided, describing the performance of the system as a function of this limiting aperture.

\section{FOURIER AND OPTICAL SYSTEMS}

From a Fourier optics perspective ${ }^{10,11}$ we may consider the optical systems in which we are interested as composed of two specific elements: propagation in air (or free-space) and propagation through lenses. In the case of free-space propagation the impulse response, in the Fresnel approximation, is given by,

$$
h(x, y)=\frac{e^{j k z}}{j \lambda z} \exp \left(j \frac{k}{2 z}\left(x^{2}+y^{2}\right)\right)
$$

where $k$ is the wave number, $k=2 \pi / \lambda$, with $\lambda$ the light wavelength. In the case of a lens, it produces the change in the curvature of the incoming beam thus generating an spherical wavefront. In the case of a lens with a focal $f$ the effect on the phase of the incoming wavefront is represented by,

$$
t_{L}(x, y)=\exp \left(-j \frac{\pi}{\lambda f}\left(x^{2}+y^{2}\right)\right)
$$

where, as in Eq. (1), we have a parabolic expression. This is a very good approximation as long as we move in the paraxial regime, i.e. not very large numerical apertures.

Let us introduce the following definitions to facilitate and to make more readable the calculations we will do in the next section:

$$
\psi\left(x, y ; Z=\frac{1}{z}\right) \equiv \exp \left(j \frac{\pi Z}{\lambda}\left(x^{2}+y^{2}\right)\right)
$$

Now we write respectively the expressions for the impulse response, Eq. (1), and lens transmission, Eq. (2), as follows:

$$
\begin{gathered}
h(x, y)=\frac{e^{j k z}}{j \lambda z} \exp \left(j \frac{k}{2 z}\left(x^{2}+y^{2}\right)\right)=\frac{e^{j k z}}{j \lambda} Z \psi(x, y ; Z)=C_{z} Z \psi(x, y ; Z) \\
t_{L}(x, y)=\exp \left(-j \frac{\pi}{\lambda f}\left(x^{2}+y^{2}\right)\right)=\psi(x, y ;-F)
\end{gathered}
$$

Previous expressions verify a series of very useful properties which may be found in the book of A. VanderLugt ${ }^{11}$. We find appropriate to list them for self containment of the paper since we make intensive use of them in the theoretical calculations in the following sections:

$\left.1^{\circ}\right) \psi(x, y ; Z)=\psi^{*}(x, y ;-Z)$

$\left.2^{\circ}\right) \psi(-x,-y ; Z)=\psi(x, y ; Z)$ 
$\left.3^{\circ}\right) \psi\left(x, y ; Z_{1}\right) \psi\left(x, y ; Z_{2}\right)=\psi\left(x, y ; Z_{1}+Z_{2}\right)$

$\left.4^{\circ}\right) \psi\left(x, y ; Z_{1}\right) \psi^{*}\left(x, y ; Z_{2}\right)=\psi\left(x, y ; Z_{1}-Z_{2}\right)=\psi^{*}\left(x, y ; Z_{2}-Z_{1}\right)$

$\left.5^{\circ}\right) \psi(c x, c y ; Z)=\psi\left(x, y ; c^{2} Z\right)$

$\left.6^{\circ}\right) \psi(x-u, y-v ; Z)=\psi(u, v ; Z) \psi(x, y ; Z) \exp \left(-j \frac{2 \pi}{\lambda z}(u x+v y)\right)$

$\left.7^{\circ}\right) \lim _{Z \rightarrow 0} \psi^{*}(x, y ; Z)=1$

$\left.8^{\circ}\right) \lim _{Z \rightarrow \infty} Z \psi(x, y ; Z)=\delta(x, y)$

$\left.9^{\circ}\right) \int_{-\infty-\infty}^{+\infty+\infty} \psi\left(x, y ; Z_{1}\right) \exp \left(-j \frac{2 \pi}{\lambda z_{2}}(u x+v y)\right) d x d y=\frac{j \lambda}{Z_{1}} \psi^{*}\left(u, v ; \frac{Z_{2}^{2}}{Z_{1}}\right)$

$\int_{-\infty}^{+\infty+\infty} \int^{*} \psi^{*}\left(x, y ; Z_{1}\right) \exp \left(-j \frac{2 \pi}{\lambda z_{2}}(u x+v y)\right) d x d y=\frac{-j \lambda}{Z_{1}} \psi\left(u, v ; \frac{Z_{2}^{2}}{Z_{1}}\right)$

\section{HOLOGRAPHIC MEMORY BASED ON A CONVERGENT CORRELATOR ARCHITECTURE}

In Fig. 1 we show the generic scheme for the experimental setup we want to model and the names for the various magnitudes and variables. We may distinguish four blocks. Two of them are before the material: object beam and reference beam. The other two are after the material (we consider transmission geometry): correlation and reconstruction subsystems. We also show the basic components in the system: beam-splitter, spatial filters, mirror, lenses, spatial light modulator (typically some liquid crystal display), storage material, and CMOS/CCD cameras. Furthermore, we should also take into account the placement of a diaphragm to limit the beam aperture incident onto the material, thus limiting the total area where actual recording of a data page takes place, enabling appropriate use of the spatial extent of the material.

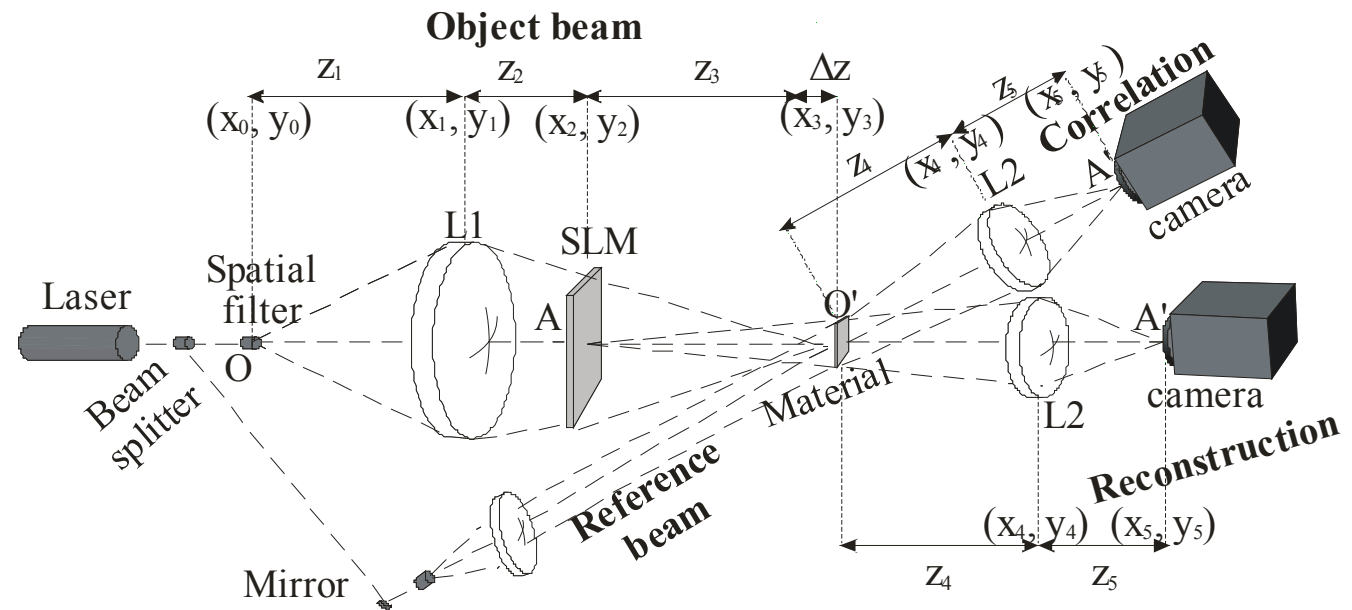

Figure 1. Scheme for the experimental setup we model in this work where the various elements and also the names for the magnitudes and variables are introduced. 


\subsection{Recording subsystem}

In Fig. 2 we show the part in the holographic memory system responsible for the storage of information. The expression for the reference beam, we consider a plane wavefront, is given by $\exp \left(j \vec{k}_{\text {ref }} \cdot \vec{r}\right)$, where $\vec{r}$ is the position vector in a certain reference system, and $\vec{k}_{r e f}$ is the wave vector indicating the direction and propagation sense for the plane wavefront. We name the object beam incident onto the material with the function $f_{3}\left(x_{3}, y_{3}\right)$. Therefore, onto the material we obtain the interference between both wavefronts. The photosensitive recording material reacts changing some property (absorption coefficient, refraction index,...) as a function of the interference pattern, given by:

$$
\mid f_{3}\left(x_{3}, y_{3}\right)+\exp \left(j \vec{k}_{r e f} \cdot \vec{r}\right)^{2}=1+f_{3}\left(x_{3}, y_{3}\right) f_{3}^{*}\left(x_{3}, y_{3}\right)+f_{3}\left(x_{3}, y_{3}\right) \exp \left(-j \vec{k}_{r e f} \cdot \vec{r}\right)+f_{3}^{*}\left(x_{3}, y_{3}\right) \exp \left(j \vec{k}_{r e f} \cdot \vec{r}\right)
$$

asterisk meaning complex conjugate. From the four summands, the last two are the interferential terms responsible for the reconstruction ( $3^{\text {rd }}$ summand) and associative search (4rth summand).

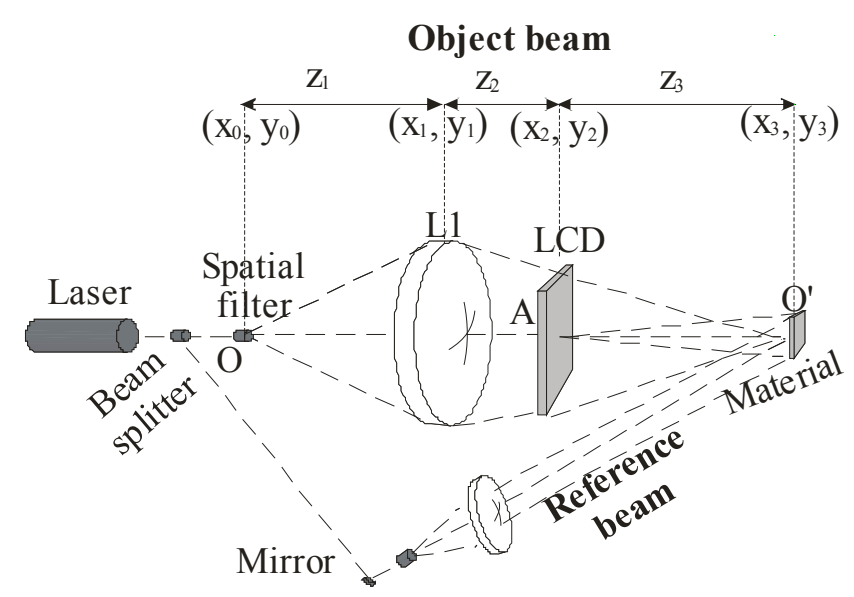

Figure 2. Scheme for the recording subsystem in the experimental setup, responsible for the storage of the data pages.

Next, we calculate the expression $f_{3}\left(x_{3}, y_{3}\right)$ for the object beam incident onto the material. Taking into account freespace propagation and transmission through lens L1, we can write, apart from some constant factor, the propagation from plane $\mathrm{O}$ to $\mathrm{O}$ ' as follows,

$$
\left.\left.f_{3}\left(x_{3}, y_{3}\right)=\llbracket\left[\psi\left(x_{1}, y_{1} ; Z_{1}\right) \psi^{*}\left(x_{1}, y_{1} ; F_{1}\right)\right] \otimes \psi\left(x_{2}, y_{2} ; Z_{2}\right)\right] f\left(x_{2}, y_{2}\right)\right] \otimes \psi\left(x_{3}, y_{3} ; Z_{3}\right)
$$

which can be expanded as,

$$
f_{3}\left(x_{3}, y_{3}\right)=\iiint_{P 1} \int_{P 2} \psi\left(x_{1}, y_{1} ; Z_{1}\right) \psi^{*}\left(x_{1}, y_{1} ; F_{1}\right) \psi\left(x_{2}-x_{1}, y_{2}-y_{1} ; Z_{2}\right) f\left(x_{2}, y_{2}\right) \psi\left(x_{3}-x_{2}, y_{3}-y_{2} ; Z_{3}\right) d x_{1} d y_{1} d x_{2} d y_{2}
$$

Applying consecutively properties 6, 3 and 4 we obtain,

$$
\begin{gathered}
f_{3}\left(x_{3}, y_{3}\right)=\psi\left(x_{3}, y_{3} ; Z_{3}\right) \iint_{P 1} \iint_{P 2} \psi\left(x_{2}, y_{2} ; Z_{2}+Z_{3}\right) f\left(x_{2}, y_{2}\right) \psi\left(x_{1}, y_{1} ; Z_{1}+Z_{2}-F_{1}\right) \ldots \\
\ldots \exp \left(-j \frac{2 \pi}{\lambda z_{2}}\left(x_{2} x_{1}+y_{2} y_{1}\right)\right) \exp \left(-j \frac{2 \pi}{\lambda z_{3}}\left(x_{3} x_{2}+y_{3} y_{2}\right)\right) d x_{1} d y_{1} d x_{2} d y_{2}
\end{gathered}
$$

Then, applying property 9 , first integrating with respect to $\left(x_{1}, y_{1}\right)$,

$$
\iint_{P 1} \psi\left(x_{1}, y_{1} ; Z_{1}+Z_{2}-F_{1}\right) \exp \left(-j \frac{2 \pi}{\lambda z_{2}}\left(x_{2} x_{1}+y_{2} y_{1}\right)\right) d x_{1} d y_{1}=\frac{j \lambda}{Z_{1}+Z_{2}-F_{1}} \psi^{*}\left(x_{2}, y_{2} ; \frac{Z_{2}^{2}}{Z_{1}+Z_{2}-F_{1}}\right)
$$


Substitution of Eq. (10) into Eq. (9) and further application of properties 3 and 4 leads to,

$$
f_{3}\left(x_{3}, y_{3}\right)=\psi\left(x_{3}, y_{3} ; Z_{3}\right) \iint_{P 2} \psi\left(x_{2}, y_{2} ; Z_{2}+Z_{3}-\frac{Z_{2}^{2}}{Z_{1}+Z_{2}-F_{1}}\right) f\left(x_{2}, y_{2}\right) \exp \left(-j \frac{2 \pi}{\lambda z_{3}}\left(x_{3} x_{2}+y_{3} y_{2}\right)\right) d x_{2} d y_{2}
$$

In the case when output plane $\mathrm{P} 3$ corresponds to the image of the initial plane $\mathrm{P} 0$, we have that the imaging equation for lens L1 is fulfilled and,

$$
Z_{2}+Z_{3}-\frac{Z_{2}^{2}}{Z_{1}+Z_{2}-F_{1}}=0
$$

Then, applying property 7 we obtain,

$$
f_{3}\left(x_{3}, y_{3}\right)=\psi\left(x_{3}, y_{3} ; Z_{3}\right) \iint_{P 2} f\left(x_{2}, y_{2}\right) \exp \left(-j \frac{2 \pi}{\lambda z_{3}}\left(x_{3} x_{2}+y_{3} y_{2}\right)\right) d x_{2} d y_{2}
$$

As a result, we obtain that $f_{3}\left(x_{3}, y_{3}\right)$ corresponds to the Fourier transform of the data page scaled as a function of the wavelength $\lambda$ and the distance between SLM and plane P3,

$$
f_{3}\left(x_{3}, y_{3}\right)=\psi\left(x_{3}, y_{3} ; Z_{3}\right) F\left(\frac{x_{3}}{\lambda z_{3}}, \frac{y_{3}}{\lambda z_{3}}\right)
$$

The quadratic phase factor influences the position of the exit plane in case there are more elements after plane P3. If not it may be dropped. In these calculations we have not included that before the material a stop is located to limit the aperture of recording area on the material. We take into account the finite size of this aperture, and the effects it introduces, in the next calculations leading to the correspondent expressions for the reconstruction process. It should be noted that in the case of the 4-f system, there is no quadratic phase factor. However, as we will see this is of no importance in our application. Let us note that in the case of 4-f systems the scale in the Fourier transform would depend on the focal length $f_{1}$ instead of on a flexible parameter, $z_{3}$. This means, that in the present case we can increase the areal density simply by diminishing the size of the Fourier transform approaching the data page to the plane P3. Eventually, we note that the present derivation is valid independent of the codification ${ }^{12-17}$ (binary intensity, binary phase, hybrid ternary modulation,...) considered for the data page.

\subsection{Reconstruction subsystem}

Let us illuminate the memory with a reconstruction beam, equal to the reference beam $\exp \left(j \vec{k}_{\text {ref }} \cdot \vec{r}\right)$ used in the recording process for one of the data pages (situation presented in Fig. 3). We take into account that before the material there is a finite aperture stop, function $p_{3}\left(x_{3}, y_{3}\right)$, as commented at the end of the previous subsection.

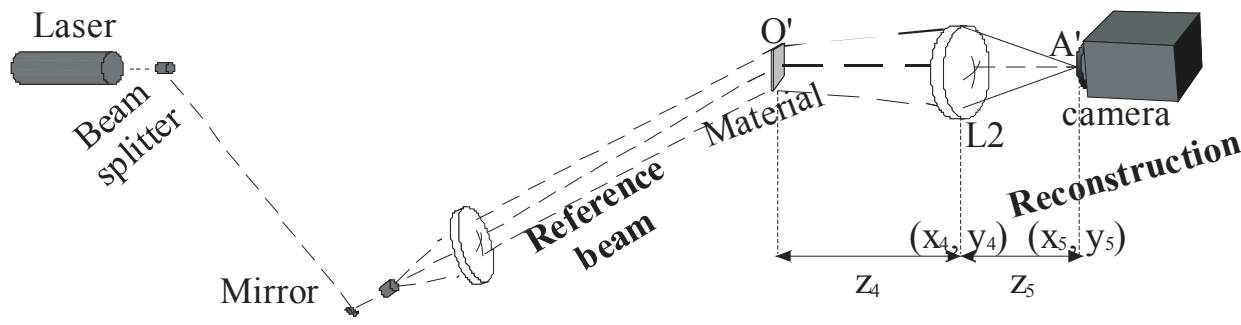

Figure 3. Scheme for the reconstruction subsystem in the experimental setup.

When illuminating the holographic memory, taking into account the interference pattern stored, described in Eq. (6), various diffraction terms may arise. From these terms we are basically interested in the ones resulting from the third and 
fourth terms in Eq. (6). Due to the properties of volume holography, it can be assumed that we basically reconstruct the object beam (third term), that is the wavefront described by $f_{3}\left(x_{3}, y_{3}\right) p_{3}\left(x_{3}, y_{3}\right)$, where we take into account the finite apertura of the stop located before the material during the recording process.

Next, we calculate the result obtained in the final plane P5, where the camera is located. The sequence followed by the wavefront from plane P3 to plane P5 can be expressed, dropping constant factors, as follows,

$$
\left.\left.f_{5}\left(x_{5}, y_{5}\right)=\llbracket\left[f_{3}\left(x_{3}, y_{3}\right) p_{3}\left(x_{3}, y_{3}\right) \otimes \psi\left(x_{4}, y_{4} ; Z_{4}\right)\right] \psi^{*}\left(x_{4}, y_{4} ; F_{2}\right)\right] \otimes \psi\left(x_{5}, y_{5} ; Z_{5}\right)\right]
$$

It can be expanded as the following integral,

$$
\begin{array}{r}
f_{5}\left(x_{5}, y_{5}\right)=\iiint_{P 3} \int_{P 4} f_{3}\left(x_{3}, y_{3}\right) p_{3}\left(x_{3}, y_{3}\right) \psi\left(x_{4}-x_{3}, y_{4}-y_{3} ; Z_{4}\right) \psi^{*}\left(x_{4}, y_{4} ; F_{2}\right) \psi\left(x_{5}-x_{4}, y_{5}-y_{4} ; Z_{5}\right) d x_{3} d y_{3} d x_{4} d y_{4} \\
f_{3}\left(x_{3}, y_{3}\right)=\psi\left(x_{3}, y_{3} ; Z_{3}\right) F\left(\frac{x_{3}}{\lambda z_{3}}, \frac{y_{3}}{\lambda z_{3}}\right)
\end{array}
$$

Where $f_{3}\left(x_{3}, y_{3}\right)$ corresponds to the object wavefront stored, Eq. (14). When substituted in Eq. (16) it results,

$$
f_{5}\left(x_{5}, y_{5}\right)=\iint_{P 3} \int_{P 4} \psi\left(x_{3}, y_{3} ; Z_{3}\right) F\left(\frac{Z_{3} x_{3}}{\lambda}, \frac{Z_{3} y_{3}}{\lambda}\right) p_{3}\left(x_{3}, y_{3}\right) \psi\left(x_{4}-x_{3}, y_{4}-y_{3} ; Z_{4}\right) \psi^{*}\left(x_{4}, y_{4} ; F_{2}\right) \psi\left(x_{5}-x_{4}, y_{5}-y_{4} ; Z_{5}\right) d x_{3} d y_{3} d x_{4} d y_{4}
$$

After applying property 6 , then properties 3 and 4, and regrouping crossed terms we obtain,

$$
f_{5}\left(x_{5}, y_{5}\right)=\psi\left(x_{5}, y_{5} ; Z_{5}\right) \iiint_{P 3} \int_{P 4} \psi\left(x_{3}, y_{3} ; Z_{3}+Z_{4}\right)\left\{\left(\frac{Z_{3} x_{3}}{\lambda}, \frac{Z_{3} y_{3}}{\lambda}\right) p_{3}\left(x_{3}, y_{3}\right) \psi\left(x_{4}, y_{4} ; Z_{4}+Z_{5}-F_{2}\right) \exp -j \frac{2 \pi}{\lambda_{4}}\left(\left(x_{3}+\frac{z_{4} x_{5}}{z_{5}}\right) x_{4}+\left(y_{3}+\frac{z_{4} y_{5}}{z_{5}}\right) y_{4}\right)\right) d x_{3} d y_{3} d x_{4} d y_{4}(18)
$$

Applying property 9, integrating with respect to plane $\mathrm{P} 4$, and then applying property 6 , it results a Fourier transform with respect to plane $\mathrm{P} 3$,

$$
\begin{aligned}
f_{5}\left(x_{5}, y_{5}\right)=\psi\left(x_{5}, y_{5} ; Z_{5}\right) \iint_{P 3} \psi\left(x_{3}, y_{3} ; Z_{3}+Z_{4}\right) F\left(\frac{Z_{3} x_{3}}{\lambda}, \frac{Z_{3} y_{3}}{\lambda}\right) p_{3}\left(x_{3}, y_{3}\right) \psi^{*}\left(x_{3}, y_{3} ; \frac{Z_{4}^{2}}{Z_{4}+Z_{5}-F_{2}}\right) \ldots \\
. . \psi^{*}\left(\frac{Z_{5} x_{5}}{Z_{4}}, \frac{Z_{5} y_{5}}{Z_{4}} ; \frac{Z_{4}^{2}}{Z_{4}+Z_{5}-F_{2}}\right) \exp \left(-j \frac{2 \pi}{\lambda} \frac{Z_{4}^{2}}{Z_{4}+Z_{5}-F_{2}}\left(\frac{Z_{5} x_{5} x_{3}}{Z_{4}}+\frac{Z_{5} y_{5} y_{3}}{Z_{4}}\right)\right) d x_{3} d y_{3}
\end{aligned}
$$

Regrouping crossed terms, then applying properties 3 and 4, and simplifying,

$$
\left.f_{5}\left(x_{5}, y_{5}\right)=\psi\left(x_{5}, y_{5} ; Z_{5}-\frac{Z_{5}^{2}}{Z_{4}+Z_{5}-F_{2}}\right) \iint_{P 3} F\left(\frac{Z_{3} x_{3}}{\lambda}, \frac{Z_{3} y_{3}}{\lambda}\right) p_{3}\left(x_{3}, y_{3}\right) \psi\left(x_{3}, y_{3} ; Z_{3}+Z_{4}-\frac{Z_{4}^{2}}{Z_{4}+Z_{5}-F_{2}}\right) \exp -j \frac{2 \pi}{\lambda} \frac{Z_{4} Z_{5}}{Z_{4}+Z_{5}-F_{2}}\left(x_{5} x_{3}+y_{5} y_{3}\right)\right) d x_{5} d y_{3}
$$

If plane $\mathrm{P} 5$ is the image of plane $\mathrm{P} 2$, then,

$$
Z_{3}+Z_{4}-\frac{Z_{4}^{2}}{Z_{4}+Z_{5}-F_{2}}=0
$$

and Eq. (20) becomes,

$$
f_{5}\left(x_{5}, y_{5}\right)=\psi\left(x_{5}, y_{5} ; Z_{5}-\frac{Z_{5}^{2}}{Z_{4}+Z_{5}-F_{2}}\right) \iint_{P 3} F\left(\frac{Z_{3} x_{3}}{\lambda}, \frac{Z_{3} y_{3}}{\lambda}\right) p_{3}\left(x_{3}, y_{3}\right) \exp \left(-j \frac{2 \pi}{\lambda} \frac{Z_{4} Z_{5}}{Z_{4}+Z_{5}-F_{2}}\left(x_{5} x_{3}+y_{5} y_{3}\right)\right) d x_{3} d y_{3}
$$

The quadratic phase factor in front of the integral may be dropped when only the amplitude of the wavefront is of interest as it is normally the case. If the data page is codified as a phase-only function, e.g. binary or multinary phase ${ }^{16,17}$, then, depending on the detection method used in plane P5, we may leave the quadratic phase term. Eq. (22) can be also expressed in a more compact form as, 


$$
f_{5}\left(x_{5}, y_{5}\right)=\psi\left(x_{5}, y_{5} ; Z_{5}-\frac{Z_{5}{ }^{2}}{Z_{4}+Z_{5}-F_{2}}\right) F T\left\{F\left(\frac{Z_{3} x_{3}}{\lambda}, \frac{Z_{3} y_{3}}{\lambda}\right) p_{3}\left(x_{3}, y_{3}\right)\right\}_{u=\frac{Z_{4} Z_{5} x_{5}}{\lambda\left(Z_{4}+Z_{5}-F_{2}\right)}, v=\frac{Z_{4} Z_{5} y_{5}}{\lambda\left(Z_{4}+Z_{5}-F_{2}\right)}}
$$

which may be rewritten as,

$$
f_{5}\left(x_{5}, y_{5}\right)=\psi\left(x_{5}, y_{5} ; Z_{5}-\frac{Z_{5}^{2}}{Z_{4}+Z_{5}-F_{2}}\right)\left\{f\left(\frac{-\lambda u}{Z_{3}}, \frac{-\lambda v}{Z_{3}}\right) \otimes P_{3}(u, v)\right\}_{u=\frac{Z_{4} Z_{5} x_{5}}{\lambda\left(Z_{4}+Z_{5}-F_{2}\right)}, v=\frac{Z_{4} Z_{5} y_{5}}{\lambda\left(Z_{4}+Z_{5}-F_{2}\right)}}
$$

where $P_{3}(u, v)$ is the Fourier transform of $p_{3}\left(x_{3}, y_{3}\right)$. In the case when $p_{3}\left(x_{3}, y_{3}\right)=1$, i.e. ideal infinite aperture, it results in $P_{3}(u, v)=\delta(u, v)$, which is the delta Dirac function, and therefore,

$$
f_{5}\left(x_{5}, y_{5}\right)=\psi\left(x_{5}, y_{5} ; Z_{5}-\frac{Z_{5}^{2}}{Z_{4}+Z_{5}-F_{2}}\right) f\left(\frac{-Z_{4} Z_{5} x_{5}}{Z_{3}\left(Z_{4}+Z_{5}-F_{2}\right)}, \frac{-Z_{4} Z_{5} y_{5}}{Z_{3}\left(Z_{4}+Z_{5}-F_{2}\right)}\right)
$$

which is nothing but the magnified and inverted image of the data page by lens L2 in plane P2. Note that if the data page $f(x, y)$ is introduced as an amplitude-only function, then the quadratic phase term can be dropped. If the aperture $p_{3}\left(x_{3}, y_{3}\right)$ is finite, what we have is that $P_{3}(u, v)$ widens and therefore smoothes the result in the reconstruction, thus blurring the edges of the bits in the data page and increasing the probability of wrong detection of $1 \mathrm{~s}$ and $0 \mathrm{~s}$. Function $p_{3}\left(x_{3}, y_{3}\right)$ acts as a low-pass filter in the frequency domain whose aperture defines de cutoff frequency. We see that the convergent correlator architecture allows obtaining the reconstructed signal as in the case of the 4-f system. Differences may arise if the data page is introduced as a non-amplitude-only function, since in this case there is a resultant quadratic factor which does not appear in the 4-f system.

\section{NUMERICAL RESULS}

As we have shown in previous section, the convergent correlator is a valid option to substitute the 4-f processor in holographic memory setups. Next we show some numerical results dealing with the influence of the finite size of the aperture stop located before the recording material. To this goal let us find the characteristic spatial frequencies in the information transmitted from the data page. This will serve to evaluate the size of the limiting aperture adequately.

Let us consider that the bits are represented in a 2-D grid (e.g. pixelated SLM) with a pitch of length d. This means that the minimum spatial frequency that can be actually represented has a period of $2 \mathrm{~d}$, or equivalently, the sampling frequency is $f_{s}=1 / d$ and the Nyquist frequency is half the sampling frequency, i.e. $f_{N}=1 / 2 d$. In the optical setup, this means that the semiaperture of the limiting stop has to be $R_{N}=\lambda z_{3} / 2 d$ to transmit the Nyquist frequency of the data page. Due to the pixelated nature of the data page, the Nyquist frequency actually corresponds to the fundamental frequency in the signal, and the various harmonics are produced at integer values of the Nyquist frequency. Let us call $R_{N}$ the Nyquist semiaperture in the rest of the present paper.

To numerically simulate the system we use the fast Fourier transform algorithm using the MATLAB software. To accurately simulate the optical system we have to consider both oversampling and zero padding of the data page bits. Oversampling is necessary in order to simulate the existence of harmonics in the Fourier plane. Zero padding, i.e. filling with zeros the oversampled matrix, has the effect of adding interpolated values in the Fourier plane. In the next calculations we consider data pages with 256x256 bits. Oversampling by a factor of 4 produces a 1024x1024 matrix, and generates 3 harmonics, plus the fundamental frequency in the Fourier plane. This matrix has been zero padded resulting in a matrix 4096x4096, which produces 3 interpolated values between the previous samples in the Fourier plane. According to all these factors, we note that to numerically simulate the limiting stop the size in pixel number of the Nyquist radius $R_{N}$ is 512 pixels.

Next we show some simulated results. We note that we have considered the existence of Gaussian additive noise with a variance of 0.005 and mean zero, which is a good estimation of the thermal noise of one of the CCD cameras in our lab. In the reconstruction the most interesting parameter is the bit-error rate (BER) which is a good account of the fidelity of 
the data page retrieved when compared with the original. BER below $10^{-3}$ are necessary so that further reduction to practical levels is obtained through additional error correction codes.

In Fig. 4 we show one of the resulting binary intensity data pages obtained after the oversampling and zero padding processes. We consider random bits data pages with an equal number of $0 \mathrm{~s}$ and $1 \mathrm{~s}$ in the present simulation.

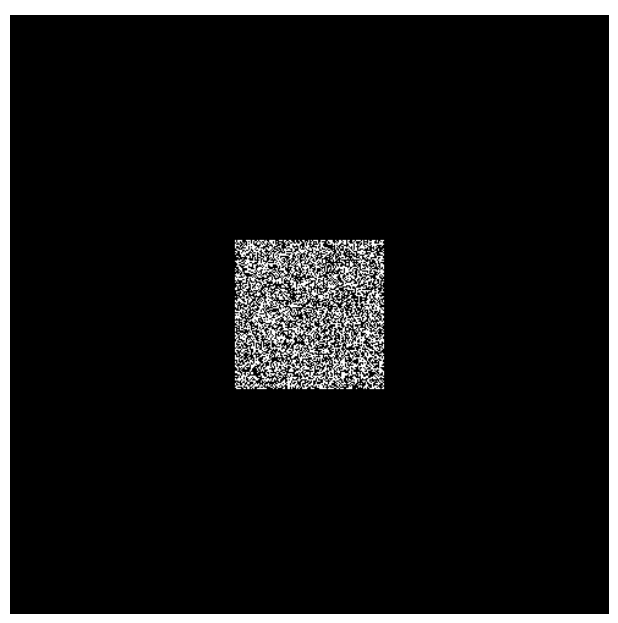

Figure 4. Binary intensity data page: 256x256 bits, oversampled a factor of 4, and with a total matrix size of 4096x4096.

In Fig. 5 we show the resulting pattern in the Fourier plane, when considering a limiting stop with a size double the Nyquist radius, $R_{N}=512$ pixels, that is the aperture radius is 1024 pixels, therefore it transmits both the fundamental frequency and the first harmonic for the data page. We can see, especially in the figure on the left, the much larger intensity of the zero frequency (or DC term) in comparison with the rest of the frequency components in the Fourier plane. This is what happens when using binary intensity data pages and may result in saturation of the dynamic range of the recording material in the location of the $\mathrm{DC}$ term, therefore deteriorating the data page information when retrieved.
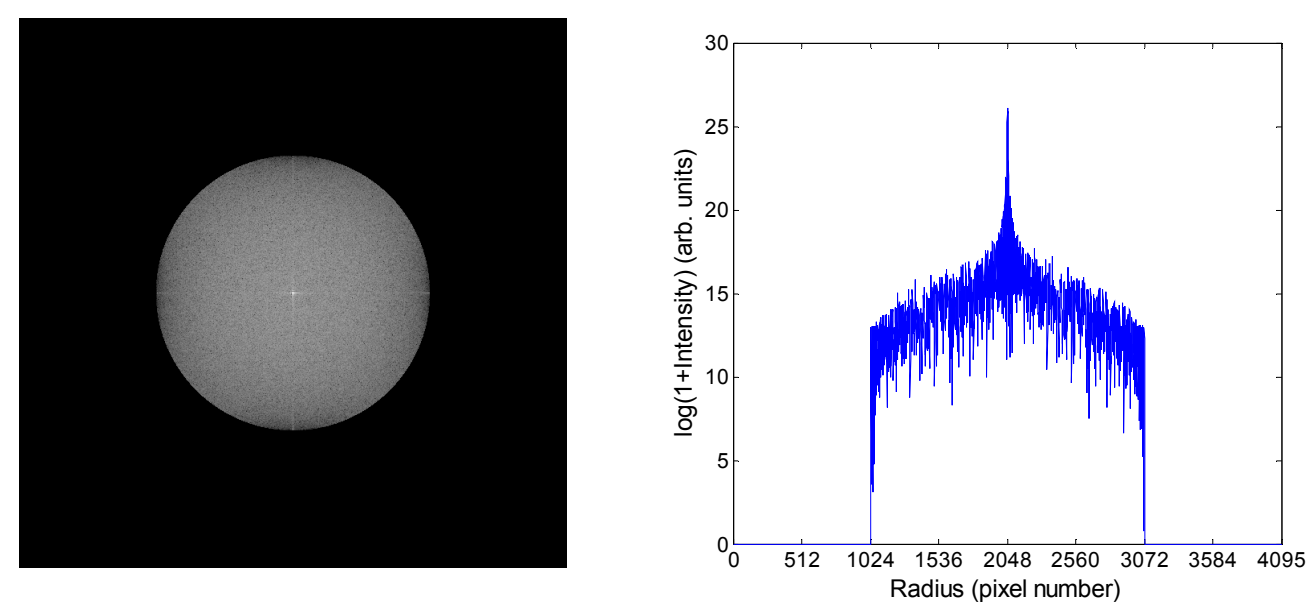

Figure 5. Fourier plane where the limiting stop is double the length of the Nyquist radius $R_{N}$, i.e. 1024. On the right the image plane, and on the left a cut across a diameter crossing the center of the Fourier plane. The representation is logarithmic. We see that the DC term is various orders of magnitude more intense that the rest of the frequency components, which is typical of binary intensity data pages. 
In Fig. 6 we show the BER obtained as a function of the aperture radius normalized by the Nyquist radius. We observe that for values about 1.6 the reconstructed data page reaches BER values better that $10^{-3}$.

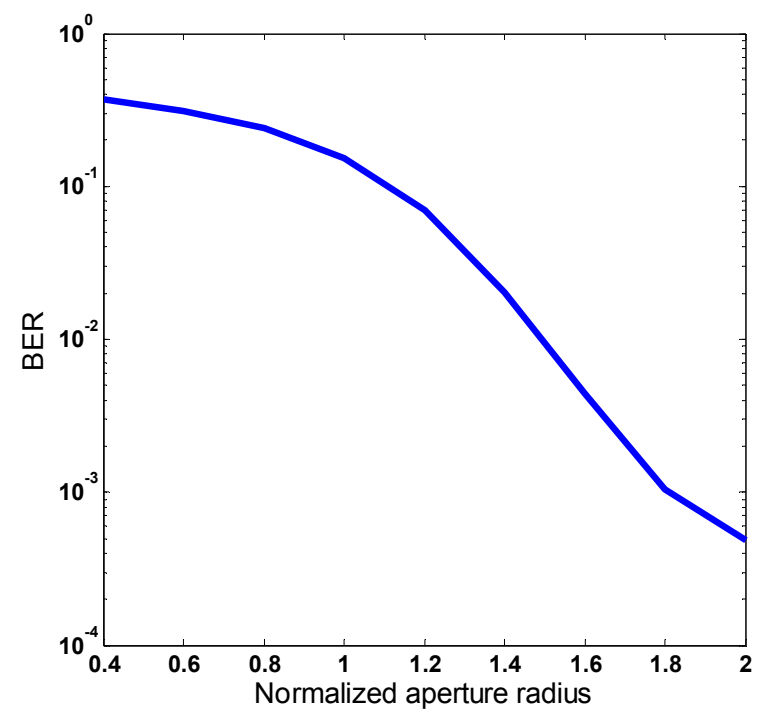

\section{CONCLUSIONS}

Typically 4-f systems are considered as the basis for holographic memory setups. However, other geometries, such as the convergent correlator, may also be considered. It allows a wider freedom in the choice of the optical systems (lenses) used in the setup since it is no longer necessary that their focal lengths match, and the total length of the setup may be shortened. We have shown in the paper, through the analytical developments, that the convergent correlator is a valid option to substitute the 4-f processor in holographic memory setups. Furthermore with this architecture the areal density can be increased simply by diminishing the size of the Fourier transform, which is accomplished approaching the data page to the plane P3. We see that the convergent correlator architecture allows obtaining the reconstructed signal at the end of the system as in the case of the 4-f system. Differences may arise if the data page is introduced as a nonamplitude-only function, since in this case there is a resultant quadratic factor which does not appear in the 4-f system. We have also done a series of numerical simulations to calculate the effect of the size of the limiting aperture, controlling the recording area on the material, on the bit-error rate in the reconstruction process. This has also served to discuss the requirements necessary for an adequate numerical simulation by means of fast Fourier transform algorithms.

\section{ACKNOWLEDGEMENTS}

This work was supported by the Ministerio de Trabajo y Competitividad of Spain under projects FIS2011-29803-C02-01 and FIS2011-29803-C02-02 and by the Generalitat Valenciana of Spain under project PROMETEO/2011/021.

\section{REFERENCES}

[1] P. J. Van Heerden, "Theory of optical information storage in solids", Applied Optics, Vol. 2, 393-400 (1963).

[2] J. Ashley, M.-P. Bernal, G. W. Burr, H. Coufal, H. Guenther, J. A. Hoffnagle, C. M. Jefferson, B. Marcus, R. M. Macfarlane, R. M. Shelby, G. T. Sincerbox, "Holographic Data Storage", IBM. J. Res. Develop. 44, 341-368 (2000). 
[3] K. Curtis, W. L. Wilson, M. C. Tackitt, A. J. Hill, and S. Campbell, "High-density, high-performance data storage via volume holography: The lucent technologies hardware platform", Holographic Data Storage 76, 359-368 (2000).

[4] K. Kincade, "Holographic data storage prepares for the real world", Laser Focus World Oct-2003, 68-73 (2003).

[5] D. Sarid and B. H. Schechtman, "A roadmap for data storage applications", Optics and Photonics News May2007, 32-37 (2007).

[6] M. Schnoes, B. Ihas, A. Hill, L. Dhar, D. Michaels, S. Setthachayanon, G. Schomberger, and W. L. Wilson, "Holographic data storage media for practical systems", in Practical Holography XVII and Holographic Materials IX, Tung H.Jeong, ed. Edts., Proc. SPIE vol. 5005, 29-37 (2003).

[7] G. T. Sincerbox, "History and physical principles", in Holographic Data Storage, H. Coufal, D. Psaltis and G. T. Sincerbox, eds., p. 3-20, Springer-Verlag, New York (2000).

[8] P. A. Mitkas and G. W. Burr, "Volume holographic optical correlators", in Holographic Data Storage, H. Coufal, D. Psaltis and G. T. Sincerbox, eds., p. 429-444, Springer-Verlag, New York (2000).

[9] G. Barbastathis and D. Psaltis, "Volume holographic multiplexing methods", in Holographic Data Storage, H. Coufal, D. Psaltis and G. T. Sincerbox, eds., p. 21-62, Springer-Verlag, New York (2000).

[10]J. W. Goodman, [Introduction to Fourier Optics], McGraw-Hill, 2nd edition, (1996).

[11]A. VanderLugt, [Optical Signal Processing], John Wiley \& Sons, (1992).

[12]J. S. Jang and D. H. Shin, "Optical representation of binary data based on both intensity and phase modulation with a twisted-nematic liquid-crystal display for holographic digital data storage", Opt. Lett. 26, 1797-1799 (2001).

[13] A. Márquez, S. Gallego, D. Mendez, M. L. Alvarez, E. Fernández, M. Ortuño, C. Neipp, A. Beléndez, and I. Pascual, "Accurate control of a liquid-crystal display to produce a homogenized Fourier transform for holographic memories", Opt. Lett. 32, 2511-2513 (2007).

[14]Z. Göröcs, E. Erdei, T. Sarkadi, F. Ujhelyi, J. Reményi, P. Koppa, and E. Lorincz, "Hybrid multinary modulation using a phase modulating spatial light modulator and a low-pass spatial filter", Opt. Lett. 32, 23362338 (2007).

[15]D. Bhargab, V. Sunil, J. Joby, P. Senthilkumaran, and S. Kehar, "Transmission type twisted nematic liquid crystal display for three gray-level phase-modulated holographic data storage systems", Opt. Laser Eng. 47, 1150-1159 (2009).

[16]J. Joseph J, and D. Waldman, "Homogenized Fourier transform holographic data storage using phase spatial light modulators and methods for recovery of data from the phase image", Appl Opt 45, 6374-6380 (2006).

[17]D. Bhargab, J. Joby, and S. Kehar, "Phase modulated gray-scale data pages for digital holographic data storage", Opt. Comm. 282, 2147-2154 (2009).

[18]E. Fernandez, A. Marquez, S. Gallego, R. Fuentes, C. García, and I. Pascual, "Hybrid Ternary Modulation Applied to Multiplexing Holograms in Photopolymers for Data Page Storage", J. Lightwave Technol. 28, 776783 (2010). 